Nouvelles perspectives en sciences sociales

Revue internationale de systémique complexe et d'études relationnelles

\title{
Le jeu comme modèle de l'activité humaine et comme modèle en théorie de l'action conjointe en didactique. Quelques remarques
}

\section{Gérard Sensevy}

Volume 7, numéro 2, mai 2012

Sur le thème de la modélisation

URI : https://id.erudit.org/iderudit/1013056ar

DOI : https://doi.org/10.7202/1013056ar

Aller au sommaire du numéro

\section{Éditeur(s)}

Prise de parole

\section{ISSN}

1712-8307 (imprimé)

1918-7475 (numérique)

Découvrir la revue

Citer cet article

Sensevy, G. (2012). Le jeu comme modèle de l'activité humaine et comme modèle en théorie de l'action conjointe en didactique. Quelques remarques. Nouvelles perspectives en sciences sociales, 7(2), 105-132.

https://doi.org/10.7202/1013056ar

\section{Résumé de l'article}

Dans cet essai, j'envisage la notion de jeu en tant qu'elle peut fournir une structure pour modéliser l'activité humaine en général, et l'activité didactique en particulier. Je tente de montrer certains aspects de sa pertinence scientifique, telle qu'elle est utilisée au sein de la théorie de l'action conjointe en didactique. Pour cela, je présente la notion de jeu en tant qu'outil de description de la pratique, c'est-à-dire en tant qu'élément de constitution d'une théorie de la pratique. Dans cette perspective, le modèle du jeu a pour fonction de mieux comprendre et expliquer l'action humaine. Après avoir présenté certains éléments d'arrière-plan sur la base desquels la notion de jeu me paraît pouvoir être employée de manière fructueuse, je m'efforce de dissiper certaines ambiguïtés attachées à l'usage de la notion, et à clarifier ce que peut signifier l'expression " utiliser le modèle du jeu ". Je tente ensuite de donner à voir certains intérêts génériques de l'usage du modèle du jeu, avant de préciser rapidement certains aspects de cet usage par comparaison à ceux de tâche et d'activité. La conclusion se concentre sur la production de critères d'usage du modèle du jeu. 


\title{
Le jeu comme modèle de l'activité humaine et comme modèle en théorie de l'action conjointe en didactique. Quelques remarques
}

\author{
Gérard Sensevy \\ Université Rennes 2 \\ Centre de recherche sur l'éducation, \\ les apprentissages et la didactique (CRÉAD)
}

\section{Introduction}

D ans cet essai ${ }^{1}$, j'envisage la notion de jeu en tant qu'elle peut fournir une structure pour modéliser l'activité humaine en général, et l'activité didactique en particulier. Je tente de montrer certains aspects de sa pertinence scientifique, sans pour autant en proposer une théorie générale.

La notion de jeu est utilisée de manière systématique au sein de la théorie de l'action conjointe en didactique². Il ne m'est pas

Ce texte est fondé sur des recherches, mais il ne constitue pas en soi un article empirique. L'usage du pronom « je " ressortit ici au genre de l'essai.

2 Gérard Sensevy et Alain Mercier, Agir ensemble. L'action didactique conjointe du professeur et des élèves, Rennes, Presses universitaires de Rennes, 2007; Gérard Sensevy, "Overcoming Fragmentation: Towards a Joint Action Theory in Didactics ", dans Brian Hudson et Meinert A. Meyer (dir.), Beyond Fragmentation: Didactics, Learning and Teaching in Europe, Opladen and Farmington Hills, Barbara Budrich, 2011, p. 60-76; Gérard Sensevy, Le Sens du Savoir. Éléments pour une théorie de l'action conjointe en didactique, 
possible, dans le cadre de cet article, de produire en quelques mots une présentation de la Théorie de l'action conjointe en didactique (TACD). Mais cela ne me semble pas préjudiciable à la compréhension de mes propos, si l'on admet que la théorie de l'action conjointe en didactique tente de proposer une théorie de la pratique. Elle tente donc de fournir des outils de description de la pratique. Dans cette perspective, la notion de jeu constitue un tel outil, utilisé autant pour sa capacité générique à donner à voir sous une certaine description toute action humaine, que pour sa capacité spécifique à rendre compte de certains aspects saillants de l'action didactique, c'est-à-dire de toute action au sein de laquelle quelqu'un entreprend de faire apprendre "quelque chose " à quelqu'un d'autre (les deux " personnes " pouvant, dans le cas de l'autodidaxie, être confondues dans la même). L'article ne se présente donc pas une sorte de "défense et illustration" du modèle du jeu. Il entend plutôt présenter une certaine façon de concevoir le modèle du jeu pour mieux comprendre et expliquer l'action humaine ${ }^{3}$.

Dans une première section (Le modèle du jeu, éléments d'arrièreplan), je montre certains éléments d'arrière-plan sur la base desquels la notion de jeu me paraît pouvoir être employée de manière fructueuse. La deuxième section (Le jeu comme modèle) s'efforce de dissiper certaines ambiguïtés attachées à l'usage de la notion, et à clarifier ce que peut signifier l'expression « utiliser le modèle du jeu ». Dans la troisième section (Des vertus de la notion de jeu en tant que modèle), je tente de donner à voir certains intérêts de l'usage du modèle du jeu. La dernière section de l'article (Jeu, activité, tâche) précise rapidement certains aspects de cet usage par comparaison à ceux de tâche et d'activité. La conclusion se concentre sur la production de critères d'usage du modèle du jeu.

J'intègrerai dans la discussion quelques références à la TACD. En effet, cette théorie s'appuie sur le postulat anthropologique

Bruxelles, De Boeck, 2011; Brigitte Gruson, Dominique Forest et Monique Loquet, Jeux de savoir, Rennes, Presses universitaires de Rennes, 2012.

3 Il faut donc saisir, ici, que l'idée de jeu n'est pas perçue dans ses dimensions " ludiques ", ou " mondaines », mais en tant que structure de modèle. 
selon lequel un très grand nombre de nos actions signifiantes est organiquement conjoint ${ }^{4}$. En ce sens, la manière dont elle traite les faits sociaux pourrait constituer dans certains cas des exemplaires $^{5}$ pour l'analyse, ce que j'essaie d'illustrer à certains moments dans ce qui suit. Ainsi, le modèle du jeu, spécifié au particulier «didactique » de la TACD, pourrait en retour contribuer à informer une analyse générique de l'action humain, qui rende compte d'une manière renouvelée de certains aspects " coopératifs" de l'action humaine.

\section{Le modèle du jeu : éléments d'arrière-plan}

L'usage générique auquel je réfere ci-dessus renvoie à l'utilisation de la notion de jeu comme un modèle, qui fait voir l'activité humaine comme un jeu. Énoncer une telle phrase, cela signifie nécessairement devoir situer l'arrière-plan à partir duquel on peut lui donner un sens. C'est ce que je vais tenter de faire.

La notion de jeu sur laquelle la TACD s'appuie est en relation étroite avec la manière dont Wittgenstein l'utilise dans ses propres analyses, en particulier lorsqu'il produit la notion de jeu de langage. La première fonction du modèle du jeu, du voircomme ${ }^{6}$ qu'il institue, s'établit donc dans une perspective grammaticale. Vincent Descombes a fourni une description éclairante de ce terme :

Qu'est-ce qui nous rend capable de suivre une ligne droite ou de tracer une ligne droite?... Ce n'est pas une question de psychologie - à quel âge et en mobilisant quelles ressources? - ni une question de philosophie transcendantale - que doit être le monde pour que je puisse m’y diriger en ligne droite? -, mais une question grammaticale ${ }^{7}$ : dans quel contexte y a-t-il un sens à dire que quelqu'un se dirige en ligne droite?

4 John Dewey, Logique. Théorie de l'enquête, Paris, Presses universitaires de France, 1993; Georges, H. Mead, L'Esprit, le soi et la société, Paris, Presses universitaires de France, 2006; Michael Tomasello, Pourquoi nous coopérons, Rennes, Presses universitaires de Rennes, 2012.

5 Thomas Kuhn, La Tension essentielle: tradition et changement dans les sciences, Paris, Gallimard, 1990.

6 Ludwig Wittgenstein, Recherches philosophiques, Paris, Gallimard, 2004.

7 C'est moi qui souligne.

8 Vincent Descombes, Le Complément de sujet, Paris, Gallimard, 2004, p. 444. 
Utiliser le modèle du jeu, c'est donc, grâce à ce modèle, tenter d'identifier la logique d'action des individus, les nécessités qu'ils doivent prendre en compte, le contexte qui donne un sens précis à leur action.

D'une certaine manière, le modèle du jeu fournit à l'analyste une première topique ${ }^{9}$ fondamentale, qu'on pourrait résumer dans des questions du type "cet individu (ces individus), à quel jeu joue-t-il? (jouent-ils)?».

On trouvera une sorte de matrice fondamentale de ce questionnement dans la structure suivante.

i) On décrit "de l'extérieur ", d'une manière " mince " pour reprendre l'opposition séminale de Gilbert Ryle ${ }^{10}$ entre description mince et description dense (épaisse), un comportement (par exemple : une personne court sur une prairie le long d'une ligne blanche, elle met le pied sur la ligne blanche, elle s'arrête de courir).

ii) Puis on rend intelligible cette action au moyen d'un certain type de description " dense ", qui répond à la question «à quoi joue-t-il? ». La description mince devient la description dense suivante : il joue au rugby. Il s'agit d'un joueur de rugby qui a mis le pied en touche. En disant « il joue au rugby ", en fournissant ce descripteur dense obtenu par la reconnaissance du jeu joué et par sa dénomination, on éclaire son action, on en fait saisir la grammaire, la structure logique (perspective grammaticale), et les nécessités qui la structurent.

Cet usage wittgensteinien du jeu se distingue sans doute de beaucoup d'autres. Par exemple, comme j'en reprendrai l'idée ci-dessous, il suit Hintikka ${ }^{11}$, qui déclare que, contrairement à ce que semblent penser nombre d'exégètes de Wittgenstein, la

$9 \quad$ Sur la notion de topique, cf. Paul Veyne, Comment on écrit L'histoire, Paris, Seuil, 1979.

10 Gilbert Ryle, Logic Lane. Collected Essays, volume 2, Londres, Routledge, 2009.

11 Jaako Hintikka, Fondements d'une théorie du langage, Paris, Presses universitaires de France, 1994. 
question essentielle autour de laquelle tourne sa "seconde " philosophie n'est pas celle des règles, mais celle des jeux, ce qui suppose, nous dit Hintikka, de substituer à la centration sur la règle une centration sur les jeux. J'ajoute que la raison de cette substitution, selon moi, tient principalement au fait que parler de "règle sans jeu » opère une réduction néfaste sur la pratique, en ce qu'elle affaisse la grammaticalité de la règle. Il suffit de reprendre l'exemple ci-dessus pour le concevoir : quel sens pourrait avoir une explication du type a) si la personne qui courait sur une prairie le long d'une ligne blanche s'est arrêtée de courir parce quelle a mis le pied sur la ligne blanche; b) c'est parce quielle a enfreint la règle de la touche", si cette explication ne renvoyait pas au jeu (ici, le rugby) dont cette règle (la règle de la touche) est une des règles définitoires?

La conception de la règle défendue par Laugier et Chauviré ${ }^{12}$ me semble du même type, puisqu'elle renvoie à l'idée que comprendre comment quelqu'un "suit une règle ", c'est d'abord comprendre quelle place la règle tient dans sa vie pratique, cette compréhension renvoyant à la praxis, à la forme de vie qui donne son sens à la règle. Le modèle du jeu constitue précisément un moyen d'identifier la praxis sur l'arrière-plan de laquelle les comportements des individus sont intelligibles.

Un autre élément d'arrière-plan pour saisir le modèle du jeu sous la description développée dans cet article tient dans son usage au sein de la sociologie de Bourdieu :

L'image du jeu est sans doute la moins mauvaise pour évoquer les choses sociales. Pourtant, elle comporte des dangers. En effet, parler de jeu, c'est suggérer qu'il y a au commencement du jeu un inventeur du jeu, un nomothète, qui a posé les règles, instauré un contrat social. Plus grave, c'est suggérer qu'il existe des règles du jeu, c'est-à-dire des normes explicites, le plus souvent écrites, etc.; alors qu'en réalité c'est beaucoup plus compliqué. On peut parler de jeu pour dire qu'un ensemble de gens participent à une activité réglée, une activité qui, sans être le produit de l'obéissance à des règles, obéit à certaines régularités. Le jeu est le lieu d'une nécessité immanente, qui est en même temps une logique

$12 \quad$ Sandra Laugier et Christiane, Chauviré, Lire les recherches philosophiques de Wittgenstein, Paris, Vrin, 2006. 
immanente. On n'y fait pas n'importe quoi impunément. Et le sens du jeu $^{13}$, qui contribue à cette nécessité et à cette logique, est une forme de connaissance de cette nécessité et de cette logique. ${ }^{14}$

Les mises en garde de Bourdieu sont précieuses : les régularités de la pratique ne sont pas issues de "l'obéissance à des règles ", mais ces régularités n'en fournissent pas moins des "nécessités immanentes ", qui déterminent la logique (de la) pratique.

Contre certains tropismes structuralistes, le modèle du jeu ajoute à la description grammaticale une prise en compte du sens de l'action pour l'acteur, de son "sens du jeu ", spécifiquement à travers la notion de stratégie (éventuellement inconsciente, l'idée de stratégie inconsciente ne constituant nullement un oxymore, comme l'a montré Bourdieu lui-même ${ }^{15}$. La notion de jeu fournit donc à Bourdieu un moyen de structurer sa description du social, mais cette opération de structuration est d'un bout à l'autre fonctionnaliste. En effet, elle renvoie à la fois à la téléologie fondamentale de l'action humaine (le sens du jeu et ses stratégies) et aux structures institutionnelles dans lequel s'exprime ce jeu (avec la notion de capital, la dialectique du champ et de l'habitus, etc.) - on pourrait d'ailleurs dire : les structures institutionnelles dont ce jeu est l'expression.

Il faut noter, pour en terminer avec cette détermination rapide d'éléments d'arrière-plan, comment la délimitation grammaticale d'espaces logiques spécifiques dans l'activité, que permet la notion de jeu, rencontre certaines des préoccupations de l'interactionnisme symbolique ${ }^{16}$. Lorsque Mead ou Blumer décrivent l'activité conjointe des individus, ils l'indexent systématiquement à des situations organisées du monde social qu'on peut tout à fait

13 Dans cette perspective, l'idée de "sens du jeu " est à rapprocher de celle de " connoisseurship » utilisée dans la littérature anglo-saxonne. Le connaisseur d'une pratique, c'est quelqu'un pourvu d'un "sens du jeu " relatif à cette pratique (cf. Gilbert Ryle, The Concept of Mind, London, Hutchinson's University Library, 1949). Cf. ci-dessous.

14 Pierre Bourdieu, Choses dites, Paris, Minuit, 1987, p. 80-81

15 Pierre Bourdieu et Loïc J. D. Wacquant, Réponses: pour une anthropologie réflexive, Paris, Seuil, 1992.

16 Georges H. Mead, L’Esprit, le soi et la société, op. cit.; Herbert Blumer, George Herbert Mead and Human Conduct, Walnut Creek, AltaMira Press, 2004. 
décrire comme des jeux, ce que l'on peut très bien saisir grâce à la perspective ébauchée ci-dessus : parler d' "activité conjointe » signifie commencer par identifier quel jeu nous allons jouer ensemble, dans lequel et grâce auquel nous allons reconnaître les mouvements (moves) de l'autre pour ce qu'ils sont et y répondre en fonction de cette reconnaissance. La notion de jeu, telle qu' elle est utilisée ici pour décrire le monde social, peut alors se spécifier à ce que l'on pourrait appeler des " jeux coopératifs ", c'est-à-dire des jeux dans lesquels le gain au jeu dépasse la seule action d'un individu, mais dépend de la coordination de plusieurs. Le jeu didactique, dans lequel le gain au jeu (faire apprendre), pour le professeur dépend organiquement du gain au jeu (apprendre) de l'élève pourrait constituer un paradigme de tels jeux coopératifs ${ }^{17}$.

Pour terminer tout à fait ce premier travail grammatical sur la notion de jeu, je voudrais faire deux remarques, l'une en passant, l'autre fondamentale.

La première remarque, qui a pour fonction de prévenir certains malentendus, tient à la question du ludique. On peut se souvenir que l'une des utilisations de Wittgenstein, notamment dans les Recherches philosophiques, de la notion de jeu, renvoie à celle d'air

17 Ce genre de « jeu coopératif " ne renvoie pas à ce qu'on appelle classiquement " jeux coopératifs» en théorie des jeux, dans lesquels par exemple des " coalitions " de joueurs jouent les unes contre les autres. En revanche, il semble se rapprocher des signaling games (David Lewis, Convention, Oxford, Blackwell, 1965; Brian Skyrms, Evolution of the Social Contract, Cambridge, Cambridge University Press, 1996; Brian Skyrms, The Stag Hunt and the Evolution of Social Structure, Cambridge, Cambridge University Press, 2004), aujourd'hui couramment utilisés dans la biologie évolutionnaire. Cette modélisation met en présence un émetteur (sender) envoyant un message caractérisant un "état de la nature" à un receveur (receiver) qui doit produire un acte adéquat à cet état en ignorant ce qu'il est. L'émetteur comme le receveur gagnent tous deux si le receveur produit l'acte adéquat. Les jeux coopératifs dont je parle sont donc des jeux de "dépendance de gain ». Dans le cas le plus général, ces jeux mettent en présence deux joueurs (instances) A et B. Le gain d'un des deux joueurs (B) est soumis au gain de l'autre (A), le gain de $\mathrm{A}$ correspondant à la production d'un " comportement " adéquat à "l'état de la nature " à propos duquel $\mathrm{B}$ détient des informations que $\mathrm{A}$ ignore, et que $\mathrm{B}$ doit donc lui " communiquer " pour que tous deux gagnent. Certaines contraintes peuvent porter sur les " messages " que $\mathrm{B}$ envoie à $\mathrm{A}$, et sur la relation de ces messages à la fois au comportement et à l'état de la nature qui lui est relié. 
de famille. Il n'est pas possible de donner une définition logiquement fermée de la notion de jeu, mais, de proche en proche, on peut lire les différents usages du terme comme liés par un air de famille. Cela signifie que d'un usage à l'autre, certains caractères "définitoires» de la notion peuvent disparaître sans que pour cela son emploi n'en soit obéré. L'usage que j'étudie ici du modèle $\mathrm{du}$ jeu (et l'usage qu'en fait la TACD) ne connote a priori rien de ludique. Pas plus qu'il ne renvoie, pour reprendre certaines analyses de Chauvier ${ }^{18}$ au fait qu'un jeu ne serait pas sérieux, ou qu'il serait gratuit. Dans l'usage que je précise ici, le jeu fournit une structure d'appréhension de l'activité qui n'entend rien dire de particulier a priori sur le caractère "ludique », « sérieux », ou " gratuit » de l'activité.

La seconde remarque est fondamentale d'un point de vue conceptuel. Je soutiens qu'un obscurcissement considérable de la notion et de son usage dans la caractérisation du monde social tient à l'oubli de la notion de stratégie.

J'ai ainsi proposé ailleurs une ontologie tripartite à la notion de jeu. Un jeu possède des règles définitoires (qui correspondent grosso modo, dans les jeux "conventionnels", au règlement du jeu), qui peuvent souvent se ramener aux règles constitutives mises en avant par Searle ${ }^{19}$. Il nécessite des règles stratégiques, qui, comme le décrit Hintikka ${ }^{20}$ explicitent comment bien jouer au jeu (elles peuvent par exemple être transmises par un connaisseur du jeu à un moins connaisseur), et des stratégies (effectives), qui constituent pour le joueur la manière concrète d'agir dans une praxis déterminée, en révélant (plus ou moins) un certain sens du jeu. Selon moi, une ontologie qui ne différencie pas ces trois entités (règles définitoires, règles stratégiques, stratégies), qui confond par exemple, comme c'est souvent le cas me semble-t-il,

18 Stéphane Chauvier, Qu'est-ce qu'un jeu?, Paris, Vrin, 2007.

19 John Searle, "What Is an Institution? ", Journal of Institutional Economics, vol. $1, \mathrm{n}^{\circ} 1,2005$, p. 1-22.

20 Jaako Hintikka, Fondements d'une théorie du langage, Paris, Presses universitaires de France, 1994; Jaako Hintikka et Gabriel Sandu, "What Is Logic? », dans Dan Gabbay, Paul Thagard et Paul Woods, Handbook of the Philosophy of Science, volume 5, Londres, Elsevier, 2006. p. 13-38. 
règles définitoires et règles stratégiques, ou d'une manière plus générale règle et stratégie, une telle ontologie achoppera, dans la description du social, sur des apories insurmontables. Elle sera contrainte, par exemple, de voir la production de stratégies complexes comme une obéissance plus ou moins aveugle à des règles cachées. Elle s'interdira donc de comprendre le système stratégique au moyen desquels les agents jouent le jeu en actualisant ou non leurs intentions ${ }^{21}$. Pour reprendre l'exemple cité plus haut, considérons que l'identification du jeu et de ses règles définitoires permet de comprendre pourquoi le joueur de rugby qui a mis «le pied en touche " s'arrête de jouer. Mais cette seule reconnaissance ne permettra nullement de saisir pourquoi le même joueur, constatant un partenaire démarqué très loin de lui, lui a fait " une passe au pied ». Pour avoir l'intelligibilité de ce qui se passe, il faudra être suffisamment connaisseur du jeu et de ses structures stratégiques. Non seulement, d'ailleurs, le connaisseur pourra-t-il comprendre a posteriori les gestes du joueur, mais, même, dans certains cas, les anticiper (cf. ci-dessous). La distinction entre règles et stratégies permet ici une distinction fondamentale « connaître les règles du jeu », et " avoir le sens du jeu ».

\section{Le jeu comme modèle}

\subsection{Le jeu : modèle ou métaphore?}

Lorsque nous utilisons (notamment en TACD), le modèle du jeu, nous avons souvent à considérer des questions du type suivant : " vous utilisez le jeu, mais cette utilisation, est-elle (seulement) métaphorique?».

Il me semble que de telles questions peuvent se dissoudre dans une certaine conception de ce qu'est un modèle, qui écarte certaines distinctions formelles (hiérarchisées) entre ces deux termes. Je considère en effet tout modèle comme un voir-comme ${ }^{22}$,

21 Gérard Sensevy, "Patterns of Didactic Intentions. Thought Collective and Documentation Work ", dans Ghislaine Gueudet, Birgit Pepin et Luc Trouche (dir.), From Text to "Lived» Resources: Mathematics Curriculum Materials and Teacher Development, New York: Springer, 2011, p. 43-58. Ludwig Wittgenstein, Recherches philosophiques, Paris, Gallimard, 2004. 
comme le fruit d'un style de pensée ${ }^{23}$, qui façonne la perception. Pour reprendre l'analyse de Chevallard ${ }^{24}$, grâce à un modèle, je peux voir un chien comme un cylindre (et user alors du rapport surface/volume pour comprendre certains aspects de son métabolisme), ou bien je peux voir $\sqrt{2}$ comme un nombre (ce que les Grecs anciens se refusaient à faire). Il y a donc, à travers la notion de voir-comme, une parenté organique entre un modèle et une métaphore, parenté d'ailleurs remarquée et commentée, notamment par Ricoeur. Évoquant « le pouvoir pictural du langage ", Ricoeur, reprenant les analyses wittgensteiniennes du voircomme, insiste sur le fait que ce pouvoir pictural consiste "à voir un aspect » :

Dans le cas de la métaphore, dépeindre le temps sous les traits d'un mendiant, c'est voir le temps comme un mendiant : c'est ce que nous faisons quand nous lisons la métaphore; lire, c'est établir une relation telle que $\mathrm{X}$ est comme $\mathrm{Y}$ en quelques sens, mais non en tous. ${ }^{25}$

Dans le sens utilisé ci-dessus par Ricoeur, "lire " c'est tout aussi bien lire une œuvre littéraire, qu'un comportement, qu'un modèle, et la modélisation elle-même peut se comprendre comme la production d'un système symbolique qu'il faudra lire en utilisant un certain voir-comme. On retrouve ici, d'un autre point de vue, une signification commune en épistémologie : un modèle (un voir-comme, une métaphore) "réduit " le système envisagé à quelques-unes de ses caractéristiques qui font sens pour le modèle : voir le chien comme un cylindre, c'est négliger le fait qu'il aboie. Modéliser la prosodie de son aboiement, c'est vraisemblablement négliger le fait qu'il peut être vu comme un cylindre.

La réponse à la question du titre de ce paragraphe (Le jeu : modèle ou métaphore?) est donc finalement assez simple : dans l'utilisation qui peut en être faite pour comprendre le monde humain, et particulièrement en TACD, le jeu est utilisé comme

23 Fleck, Ludvick, Genèse et développement d'un fait scientifique, Paris, Les Belles lettres, 2005.

24 Yves Chevallard, "Éducation et didactique : la tension essentielle ", Éducation et didactique, vol. 1, n 1, 2007, p. 9-28.

25 Paul Ricoeur, La Métaphore vive, Paris, Seuil, 1990, p. 269. 
un modèle, un voir-comme. Nous modélisons les pratiques humaines grâce à la structure sémantique et syntaxique qu'offre le jeu en tant que modèle, ce qui signifie que nous les voyons comme des jeux, que nous les produisons et les lisons comme des jeux.

2.2. Le jeu comme modèle : le concret et l'abstrait, la réduction au particulier

Si le jeu est un modèle, il est sans doute utile de préciser dans quelle conception générale de ce qu'est un modèle on peut l'inscrire. Je suivrai ici les analyses de Nancy Cartwright ${ }^{26}$. Le modèle est le trait d'union entre une théorie (par exemple la TACD) et les domaines empiriques que la théorie s'efforce de rendre intelligibles, et qui rendent en retour la théorie, à travers les modèles, " opérationnelle ». Ainsi, le modèle est une spécification concrète de la théorie.

Cartwright s'appuie d'une manière particulièrement heuristique sur Lessing ${ }^{27}$ pour montrer que les modèles, selon elle, sont dans le même rapport à la théorie que le récit d'une Fable l'est à la morale de cette fable. Cartwright s'appuie sur une fable citée par Lessing : "Une martre dévora un coq de bruyère, un renard étrangla la martre, et un loup étrangla le renard ${ }^{28}$ " et sa morale : "Les plus faibles sont toujours proies des plus forts ". Au plan épistémologique, l'idée fondamentale de Lessing, dans la continuation de la pensée des Lumières et de Christian Wolff, est que " la connaissance intuitive » est claire en elle-même, et que la connaissance symbolique « emprunte sa clarté à [la connaissance] intuitive ${ }^{29}$. Lessing en arrive ainsi à l'argument suivant : "pour donner à une conclusion symbolique générale toute la qualité dont elle est capable...nous devons la réduire au particulier afin de la connaître intuitivement ${ }^{30} "$.

26 Nancy Cartwright, The Dappled World: A Study of the Boundaries of Science, Cambridge, Cambridge University Press, 1999.

27 Gotthold E. Lessing, Traités sur la fable, Paris, Vrin, 2008.

28 Je cite ici la traduction française de la Fable, due à Hagedorn (1738).

29 Gotthold E. Lessing est ici cité par Cartwrigh, The Dappled World, op. cit., p. 38.

$30 \quad$ Ibid., p. 38. 
Cette réduction ${ }^{31}$ au particulier ${ }^{32}$ joue un rôle tout à fait essentiel dans le mécanisme à la fois épistémologique et cognitif de la fable, comme le montre cette autre citation de Lessing travaillée par Cartwright : "le général existe seulement dans le particulier et peut seulement devenir visualisable dans le particulier $^{33} "$.

Cette relation abstrait-concret, au cour de la fable, est donc aussi, pour Cartwright, au cœur des modèles de la physique :

Considérons $\mathrm{F}=$ ma. Je prétends qu'il s'agit d'une vérité abstraite relative à des assertions à propos des positions, des mouvements, des masses, et des étendues, de la même façon que la morale de Lessing «Les plus faibles sont toujours proies des plus forts» est abstraite relativement aux plus concrètes descriptions qui l'aménagent (fit out). Être soumis à une force d'une certaine intensité, disons F, est une propriété abstraite, comme être plus faible que. La loi de Newton dit que si quelque chose a cette propriété, il en a d'autres, en particulier une masse et une accélération qui, multipliées l'une par l'autre, donnent la valeur numérique déjà mentionnée, F. C'est comme dire que quelqu'un de plus faible sera aussi une proie pour le plus fort ${ }^{34}$.

Ainsi, dans cette conception, les modèles spécifient à certains particuliers ce que les théories énoncent de manière universelle. $\mathrm{La}$ « loi » $\mathrm{F}=$ ma peut se " concrétiser » au sein de divers modèles (par exemple en électricité ou à propos du pendule). On peut reprendre alors, comme le fait Lessing dans les citations ci-dessus, la distinction leibnizienne (dont Lessing use à travers sa lecture de Christian Wolff, selon Torra-Mattenklott ${ }^{35}$ ) entre intelligence

31 On conçoit ici que cette « réduction » est loin d'une approche réductionniste. Il s'agit plutôt d'insister sur l'importance du particulier pour l'intelligibilité (de l'action humaine, notamment).

32 Gérard Sensevy, Andrée Tiberghien, Jérôme Santini, Sylvain Laubé et Peter Griggs, "An Epistemological Approach to Modelling: Cases Studies and Implications for Science Teaching ", Science Education, vol. 92, n 3, 2008, p. 424-446.

33 Gotthold E. Lessing, cité par Nancy Cartwright, The Dappled World, op. cit., p. 38.

34 Nancy Cartwright, The Dappled World..., op. cit., p. 44.

35 Catherine Torra-Mattenklott, "The Fable as Figure: Christian Wolff's Geometric Fable Theory and Its Creative Reception by Lessing and Herder ", Science in Context, vol. 18, $\mathrm{n}^{\circ} 4,2005$, p. 525-552. 
intuitive et intelligence symbolique ${ }^{36}$, pour dire que les modèles amènent à une certaine forme d'intuition (d'intelligence intuitive) ce que les théories et les lois (les " généralisations symboliques ", pour utiliser le vocabulaire de $\mathrm{Kuhn}^{37}$ ) expriment dans le symbolique (grâce à l'intelligence symbolique).

Si la notion de jeu, donc, a une fonction de modèle pour certaines théories, c'est qu'elle concrétise d'une certaine manière ces théories. En tentant de construire une réponse, à propos de l'action d'un individu, à la question "à quoi joue-t-il? ", le modèle du jeu oblige à une description spécifique de telle partie du social, à la reconnaissance des enjeux du jeu, de ses règles et stratégies, et elle oblige ainsi la théorie à se contextualiser aux particuliers qu'elle tente d'éclairer.

Par exemple, la TACD peut produire l'assertion théorique (une forme "faible " de généralisation symbolique) selon laquelle l'action didactique est comprise à travers l'action conjointe du professeur et de l'élève, mais cette assertion est censée trouver une meilleure articulation ${ }^{38}$ avec la réalité et certains de ses particuliers à travers le modèle du jeu d'apprentissage. Un tel jeu d'apprentissage caractérise le jeu du professeur sur le jeu de l'élève dans la dialectique entre les connaissances antérieures de l'élève (telles qu'elles apparaissent au sein du contrat didactique) et les capacités que l'élève doit construire (grâce à son action dans le milieu didactique).

Tout modèle, et celui du jeu comme les autres, construit alors une représentation de la réalité. On pourrait reprendre ici certaines des analyses de Van Fraassen ${ }^{39}$ pour montrer comment l'usage du modèle du jeu permet de fournir une "vue " d'une pratique humaine, une certaine forme de représentation qui la fait voir d'une manière susceptible d'identifier certaines de ses caractéristiques sans cela inaperçues.

\footnotetext{
36 Gottfried Wilhelm Leibniz, Opuscules philosophiques choisis, Paris, Vrin, 1954.

37 Thomas Kuhn, La Tension essentielle: tradition et changement dans les sciences, op. cit.

38 Ian Hacking, Concevoir et expérimenter, Paris, Christian Bourgois, 1989.

39 Baas Van Fraassen, Scientific Representation: Paradoxes of Perspective, Oxford, Oxford University Press, 2008.
} 


\section{Des vertus de la notion de jeu en tant que modèle}

Je voudrais maintenant préciser davantage en quoi le modèle du jeu peut être productif et pertinent, pour les sciences de l'homme et de la société dans leur ensemble. Je me focaliserai sur quelques aspects.

\subsection{Le modèle du jeu instaure une relation organique entre intentions des agents et structure}

Au sein des sciences de l'homme et de la société, une discussion récurrente est celle des relations entre intentions des agents et structure. On pourrait alors opposer des modèles d'abord téléologiques, qui accordent une place décisive aux intentions des agents, et des modèles d'abord structuraux, qui cherchent à déterminer avant tout la structure sociocognitive qui peut expliquer l'action. Face à ce dilemme, je soutiens que le modèle du jeu permet de penser d'une manière adéquate et originale l'agentivité. En effet, voir une activité humaine comme un jeu, c'est à la fois rendre compte des intentions des joueurs, et les reconnaître pour ce qu'elles sont en tant qu'émanations de structures qui transcendent pour une grande part les " choix subjectifs " des individus. Comme le remarquait Gallie, un connaisseur d'un jeu peut anticiper avec beaucoup de finesse et d'à propos sur le jeu lui-même et les intentions des joueurs. Gallie, en effet, dans son élucidation de la " compréhension historique ", donne une place fondamentale au récit, et au fait que cette compréhension peut se conceptualiser sous l'espèce d'un "following-a-story model ", qu'il transforme bientôt en un "following-a-game model ", à l'image des spectateurs d'une partie de cricket :

According to their level of technical knowledge, judgment and experience, different members of the group [Gallie évoque ici des spectateurs plus ou moins connaisseurs du jeu] will follow some moves with far more understanding than the majority, whilst some moves (say of captaincy or of batting or bowling tactics) will be fully appreciated only by the local expert and noticed perhaps by only a few others ${ }^{40}$.

$40 \quad$ Walter B. Gallie, Philosophy and the Historical Understanding, Londres, Chatto and Windus, 1964, p. 34. En fonction de leur niveau de connaissance 
Si le connaisseur du jeu peut anticiper, c'est parce qu'il peut/ sait lire l'action en identifiant les signes décisifs attachés aux actions des joueurs. Mais ces signes ne sont pas seulement signes des intentions et de l'action elle-même, ils sont également signes de la compréhension du jeu par l'agent, compréhension que peut partager l'analyste qui connaît bien le jeu joué.

Dans cette perspective, la citation ci-dessus saisit, me semblet-il, l'essentiel de ce que le modèle du jeu peut apporter pour comprendre ce que c'est d'être confronté à une pratique donnée - en prenant conscience que quiconque agit est confronté, pendant qu'il agit, à la pratique d'autrui. Comprendre autrui, dans ce modèle, c'est bien comprendre le jeu qu'il joue, et, si, comme l'explicite Gallie, on est " connaisseur » de la pratique, on devient capable d'apprécier des coups qu'à peine quelques-uns peuvent seulement appréhender, et qui, pour la plupart des observateurs, sont littéralement invisibles.

On pourrait d'ailleurs poursuivre cette analyse, et faire remarquer que la formation des capacités humaines pourrait s'apprécier comme une familiarisation progressive aux jeux sociaux. Les romans de formation peuvent d'ailleurs se lire dans cette conception. Pour ne prendre qu'un exemple, dans un ouvrage qui en contient beaucoup, on peut se souvenir que le jeune Marcel, dans le grand roman de Proust (À la recherche du temps perdu) est abordé à Balbec, dans la rue, par le baron de Charlus (c'est leur première rencontre), qui cherche à le séduire (on pourrait dire "à le lever »). Mais le jeune narrateur n'identifie aucun des signes émis par Charlus, il le prend pour fou. À la lettre, il ne reconnaît pas «le jeu de séduction », et sa structure propre.

Bien plus tard, le même Marcel, rompu aux jeux de séduction et conscient de la possible homosexualité des personnes qu'il côtoie, identifie sans peine le jeu du même genre auquel se livrent

technique, de leur jugement et de leur expérience, les différents membres du groupe suivront certains coups avec bien plus de compréhension que la majorité, tandis que certains autres (concernant par exemple le capitanat, le "batting ", ou les tactiques de quilles) seront pleinement appréciés uniquement par l'expert local et reconnus peut-être seulement par un petit nombre d'autres. 
Charlus et Jupien, et rapporte les signes du ballet qui les réunit aux parades nuptiales des insectes. La formation du narrateur, ici, revient à la compréhension du jeu social, à travers certains jeux sociaux particuliers, et dans la reconnaissance des systèmes de signes sur lesquels ils reposent, tout jeu conjoint (comme celui de la séduction décrit ici) impliquant une sémiose spécifique. Là où jadis il ne reconnaissait pas le jeu joué, là où donc les intentions de l'autre lui étaient inscrutables, le jeu devenu transparent lui permet de lire les intentions d'autrui et les signes qui les concrétisent. Le roman d'apprentissage, le roman de formation (Bildungsroman) peut se concevoir comme une familiarisation progressive aux jeux sous lesquels on peut décrire l'expérience humaine.

La force du modèle du jeu, ici, est bien de lier organiquement les intentions de l'agent et la structure de son action. Le modèle du jeu permet de comprendre les intentions du joueur à travers la structure et la structure du jeu à travers ses intentions. Il lie organiquement structure et agentivité.

3.2. Le modèle du jeu produit une dialectique féconde entre grammaticalité de l'action et sens de l'action pour l'acteur

Dans une telle conception, on peut se placer du point de vue du joueur, occuper ainsi, après le point de vue « externe » du paragraphe précédent, un point de vue "interne ", afin de dissoudre un dualisme funeste qui opposerait point de vue "intrinsèque " (l'action vue depuis les "préoccupations " des acteurs) et point de vue "grammatical " (l'action vue depuis les nécessités ou régularités de l'action). En effet, le modèle du jeu rend compte "par construction », j'en ai avancé l'idée, des nécessités de l'action, de sa grammaire. Mais toute personne qui joue un jeu le joue à sa manière, à sa façon, avec le sens du jeu qui lui est propre. User du modèle du jeu permet à la fois de reconnaître les nécessités immanentes à l'action même (notamment à travers l'identification des règles définitoires, des règles stratégiques, et des stratégies potentielles à la disposition des agents) et la manière 
dont tout un chacun s'exprime dans le jeu (la façon dont son idiosyncrasie l'amène à produire les stratégies qui, pour une part au moins, lui sont propres). Finalement, dans cette perspective, un jeu est une institution comme l'institution de la langue, telle qu'elle peut être pensée par Merleau-Ponty :

Dans l'acte de parler, dans son ton et dans son style, le sujet atteste son autonomie, puisque rien ne lui est plus propre, et cependant, il est au même moment et sans contradiction tourné vers la communauté linguistique et tributaire de la langue. La volonté de parler est une même chose avec la volonté d'être compris ${ }^{41}$.

Lorsque je parle, je ne peux le faire qu'en utilisant les mots et expressions d'autrui, que je n'ai pas inventés. Mais dans le même temps, j'exprime à travers ma voix ce que je suis le seul à pouvoir exprimer, et je dois apprendre, comme le montre Laugier ${ }^{42}$, à projeter les mots de la langue dans de nouveaux contextes, pour pouvoir vraiment signifier ce que je dis. Sous cette description, on peut concevoir le modèle du jeu, dans sa structure profonde, comme d'une même essence que l'institution de la langue. Le modèle du jeu permet ainsi de voir tout à la fois la pratique comme le suivi d'un usage et comme son appropriation, qui demande que je me rende capable moi-même de jouer le jeu " pour moi-même ".

3.3. Le modèle du jeu suppose la reconnaissance d'enjeux et l'identification d'un gain

Jouer à un jeu, c'est en général gagner ou perdre. Mais les enjeux du jeu, et son gain, ne se réduisent pas forcément à une performance brute. Par exemple, le gain du jeu que peut constituer la lecture d'un livre peut consister, selon le cas, à connaittre son titre, ou bien à pouvoir le commenter, ou bien à pouvoir en user comme d'une expérience pour mieux mener sa propre vie, etc. Ici, la plasticité du modèle du jeu, des enjeux qu'on peut lui afférer, et des gains au moyen desquels on peut le caractériser, cette plasticité épouse celle de l'activité humaine, et des diffé-

\footnotetext{
41 Maurice Merleau-Ponty, Éloge de la philosophie et autres essais, Paris, Gallimard, 1962, p. 56.

42 Sandra Laugier, "L'Éducation des adultes comme philosophie morale ", Education et didactique, vol. 5, n 3, 2011, p. 135-144.
} 
rentes manières de la concevoir. Pouvoir considérer une pratique humaine sous le modèle du jeu, c'est donc se donner les moyens de la conceptualiser de différentes façons, et de reconnaître les différentes téléologies qu'une pratique particulière peut impliquer. La question "à quoi joue-t-il? " se double ainsi d'une autre, " en vue de quoi joue-t-il de cette façon? ". La réponse à cette seconde question reposera elle aussi sur ce que j’ai appelé les descripteurs denses de l'action, obtenus par la reconnaissance et la dénomination du jeu. Dans l'exemple ci-dessus, il y a ainsi différentes réponses, à la fois " téléologiques " (parce qu'elles donnent le but de l'action) et "structurales » (parce qu'elles font saisir sa conformation) à la question " en vue de quoi joue-t-il de cette façon? " : " connaître le titre du livre »; " commenter le livre »; " user du livre comme d'une expérience pour mener sa propre vie».

Le fait que le modèle du jeu suppose l'identification d'enjeux et d'un gain au jeu présente un autre intérêt, qu'on pourrait probablement qualifier de pragmatiste. La considération d'une activité humaine sous la catégorie du gain qu'elle permet ou non d'atteindre, et sur lequel elle repose (pour reprendre l'exemple précédent : « je connais le titre du livre »; "j’ai commenté en connaisseur tel passage du livre »; "j'ai usé de ce livre pour mieux poser et résoudre un problème dans mon existence ») permet de distinguer entre elles les actions selon leurs conséquences pratiques. On peut rappeler ici les remarques de William James, dans son texte "What is pragmatism? " à propos de la "méthode pragmatiste " :

The pragmatic method is primarily a method of settling metaphysical disputes that otherwise might be interminable. Is the world one or many? fated or free? - material or spiritual? - here are notions either of which may or may not hold good of the world; and disputes over such notions are unending. The pragmatic method in such cases is to try to interpret each notion by tracing its respective practical consequences. What difference would it practically make to any one if this notion rather than that notion were true? If no practical difference whatever can be traced, then the alternatives mean practically the same thing, and all dispute is idle. Whenever a dispute 
is serious, we ought to be able to show some practical difference that must follow from one side or the other's being right ${ }^{43}$.

Ce que le modèle du jeu, à travers la reconnaissance des enjeux et l'identification du gain qui lui sont attachés, permet de produire, précisément, c'est bien une forme d'interprétation de l'action qui tente de " tracer ses conséquences pratiques ». On peut soutenir que les conséquences pratiques les plus fortes d'une action donnée résident dans le fait que cette action soit considérée comme satisfaite, en fonction des buts que s'est donnés celui qui l'a menée. Modéliser une action donnée comme un jeu, et identifier le gain au jeu, c'est se rendre capable de distinguer entre elles des pratiques, et ainsi atteindre, d'une certaine manière, à la vérité de ces pratiques.

3.4. Le modèle du jeu permet de penser et de décrire la différence entre soi et le contexte

Le modèle du jeu, en décrivant la grammaire d'une action, nous fait saisir comment le contexte agit sur l'action. Il peut donc nous rendre conscient de la manière dont les divers contextes que nous traversons ${ }^{44}$ peuvent façonner de multiples «identités " dont le système forme le soi $^{45}$. Le modèle du jeu peut donc constituer un outil précieux pour caractériser les contextes et les dispositions

43 William James, dans H. Standish Thayer (dir.), Pragmatism. The Classic Writings, London, Hackett, 1982, p. 210. La méthode pragmatique est d'abord une méthode de règlement de disputes métaphysiques, qui, sans cela, pourraient être interminables. Le monde est-il un ou multiple? Enchanté ou libre? Matériel ou spirituel? Chacune de ces notions peut ou non décrire le monde adéquatement, et des disputes à ce sujet sont sans fin. La méthode pragmatique pour de tels cas consiste à essayer d'interpréter chaque notion en déterminant ses conséquences pratiques. Quelle différence cela ferait-il pratiquement si l'une de ces notions plutôt qu'une autre était vraie? Si aucune différence pratique ne peut être relevée, alors les alternatives signifient pratiquement la même chose, et la dispute est sans objet. Chaque fois qu'une dispute est sérieuse, nous devons être capable de montrer quelque différence pratique qui résulte du fait qu'une alternative ou une autre est correcte.

44 Bernard Lahire, L'Homme pluriel: les ressorts de l'action, Paris, Hachette, 2007.

45 Rom Harre et Luk Van Langenhove, Positioning Theory: Moral Contexts of Intentional Action, Londres, Blackwell, 1999. 
qu'ils activent ${ }^{46}$. Il permet ainsi de construire et de structurer les différences intercontextuelles auxquelles les individus ont à faire face, du point de vue des règles définitoires des jeux par lesquels on peut modéliser l'activité en contexte, et des systèmes stratégiques qu'ils produisent au sein de ces divers jeux. Il permet aussi de comprendre comment les individus, à certains moments, peuvent subitement " changer de jeu ", ou bien comment ils peuvent "jouer un jeu contre un autre ». Il ne s'agit plus alors de jouer aussi bien qu'on sait le faire tel jeu social, mais d'abandonner le jeu qu'on est en train de jouer pour en jouer un autre, qui convient mieux aux buts que l'on se fixe.

3.5. Le modèle du jeu fournit un réseau sémantique de profondes topiques pour caractériser l'action.

Je mentionnerai enfin une dernière vertu du modèle du jeu, dans

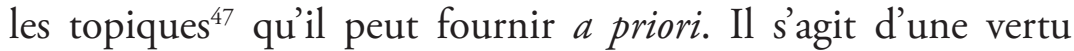
descriptive et sémantique. L'usage du terme suppose un jeu de langage qui fournit une formidable matière première à inventorier. Par exemple, ainsi que l'a montré Bourdieu ${ }^{48}$, jouer un jeu, cela suppose de se " prendre au jeu ", ce qui signifie que le modèle possède une aptitude, sise dans le langage, à décrire les émotions et les affects qui accompagnent la cognition.

Ou bien, jouer un jeu demande un certain « jeu », une certaine déformabilité dont Culioli ${ }^{49}$ a montré qu'elle est essentielle au langage, et dont je postule qu'elle est corrélativement essentielle à l'opération de modélisation en terme de jeux.

Ou encore, jouer un jeu demande à ce qu'on puisse identifier le "hors-jeu ", et, dans bien des cas, à ce qu'on soit capable de jouer à la limite du hors-jeu. Notons d'ailleurs que cette dernière topique (celle du « hors-jeu ») peut être précieuse, sous une autre

46 Bernard Lahire, La Condition littéraire. La double vie des écrivains, Paris, La Découverte, 2006; Bernard Lahire, L'Homme pluriel : les ressorts de l'action, op. cit.

47 Paul Veyne, Comment on écrit L'histoire, Paris, Seuil, 1979.

48 Pierre Bourdieu et Loïc J. D. Wacquant, op. cit.

49 Antoine Culioli, Variations sur la linguistique. Entretiens avec Frédéric Fau, Paris, Klincksieck, 2009. 
description, pour décrire les phénomènes d'exclusion. On peut, en toute connaissance de cause, jouer à la limite du hors jeu en tant que bon connaisseur du jeu. Mais on peut aussi se retrouver, dans une institution donnée, hors-jeu, parce qu'on est dépourvu du capital symbolique et/ou du capital informationnel qui permet de jouer le jeu, de participer au jeu.

\section{Jeu, activité, tâche}

J'ai essayé de le montrer ci-dessus, l'usage du modèle du jeu entend lier téléologie et structure pour retrouver la dynamique dispositionnelle ${ }^{50}$ de l'action. J'ai indiqué plus haut qu'une question souvent posée à des utilisateurs du modèle du jeu concernait l'aspect ou non " métaphorique " de l'usage du concept, et j'ai répondu en montrant l'aspect nécessairement métaphorique (au sens du voir-comme) de tout modèle. Deux autres questions peuvent être fréquemment posées à l'usage de ce modèle. La première concerne les liens entre la caractérisation en termes de jeu et la théorie de l'activité. La seconde réfère à l'éventuelle relation existant entre la notion de jeu et celle de tâche. Je vais fournir quelques éléments de réponse à ces questions.

L'usage du modèle du jeu peut très bien s'inscrire au sein de l'épistémologie illustrée par la théorie historico-culturelle de l'activité, telle qu'elle est par exemple présentée par Engeström. Celui-ci, s'intéressant à l'intentionnalité collective et à l'agentivité distribuée, distingue cinq principes pertinents pour leur étude :

1/ le principe de l'orientation/objet, 2/ le principe de la médiation par les instruments et les signes; 3 / le principe de la constitution mutuelle des actions et de l'activité; $4 /$ le principe de la déviation et des contradictions comme source de changement, et $5 /$ le principe de l'historicité. ${ }^{51}$

Sans pouvoir entrer, dans le cadre de cet article, dans la discussion de chacun de ces principes rapporté au modèle du jeu

\footnotetext{
50 Bernard Lahire, L'Homme pluriel : les ressorts de l'action, op. cit.

51 Yrjo Engeström, "L'Interactivité orientée-objet: vers une compréhension de l'intentionnalité collective dans les activités distribuées ", dans Jean-Marie Barbier et Marc Durand (dir.), Sujets, activités, environnements. Approches transverses, Paris, Presses universitaires de France, 2006, p. 145.
} 
que je présente ici, j’affirmerai une compatibilité générale des principes exprimés ci-dessus avec ce modèle, particulièrement dans le cadre de la TACD. Mais je voudrais attirer l'attention sur le fait que de tels principes réferent à un niveau très général.

La théorie de l'activité, telle qu'elle est produite dans ces principes, désigne de fait une théorie de la connaissance. Elle explicite selon moi certaines conditions auxquelles toute théorie, en sciences de l'homme de la société, doit pouvoir répondre si elle veut atteindre une certaine forme de pertinence. Au sens courant d'une théorie scientifique, donc (la théorie darwinienne de l'évolution, ou la théorie piagétienne de l'équilibration), la théorie de l'activité n'est pas une théorie, elle constitue plutôt, selon moi, un système épistémologique qui peut très utilement orienter l'élaboration des théories en sciences de l'homme et de la société. Par rapport à la théorie de l'activité, le modèle du jeu, lui, se place, pourrait-on dire, deux niveaux plus bas. Le modèle du jeu n'est pas une théorie scientifique, au sens où il ne dresse pas un système d'abstractions qualitatives ${ }^{52}$ apte à produire des connaissances sur une portion du monde, mais il est bien un modèle (au sens que j'ai essayé de décrire ci-dessus), particulièrement adapté, selon moi, à caractériser concrètement l'activité humaine, à partir d'une théorie. Peut-être faudrait-il lui donner, d'ailleurs, un statut particulier de modèle générique, au sens où il peut caractériser diverses formes de l'activité humaine.

Concernant les relations entre la notion de jeu et celle de tâche, on peut produire des considérations presque opposées. Alors que l'usage du modèle du jeu (particulièrement en TACD) trouve une compatibilité forte avec l'épistémologie (la théorie) de l'activité, il se trouve d'une certaine manière en opposition avec l'usage de la notion exclusive de tâche. Il ne s'agit pas ici de critiquer la pertinente structure (tâche/activité) commune en ergonomie, ou l'usage du terme et du concept au sein de la profonde modélisation praxéologique proposée par Chevallard ${ }^{53}$. Il s'agit plutôt de révoquer en doute la centration sur la tâche,

$52 \quad$ Ian Hacking, Concevoir et expérimenter, op. cit.

53 Yves Chevallard, "Éducation et didactique : la tension essentielle ", op. cit. 
qu'on peut trouver à la fois dans certaines recherches en éducation et dans certaines pratiques éducatives. On pourra ainsi parler systématiquement de la "tâche de l'élève ", ou évoquer la " tâche de l'enseignant ". On produira alors un rabattement de l'activité sur la tâche, d'ailleurs dénoncé depuis longtemps par les ergonomes. Tout se passe, dans les usages problématiques du terme et de la notion de tâche, comme si le professeur ou l'élève, plus généralement l'agent au sein du monde social, devait être ramené à un opérateur de tâches, ce dernier mot étant utilisé au sens du dictionnaire. Il est utile de méditer ce sens initial, avec l'exemple associé, donné par le Trésor de la langue française, définition (en substance) et exemple (à la lettre) repris du Littré: "Travail défini et limité, imposé par autrui ou par soi-même, à exécuter dans certaines conditions. Assigner, donner une tâche à des écoliers, des ouvriers".

L'usage du mot tâche, s'il n'est pas produit dans un système théorique spécifique, par exemple celui de l'ergonomie, qui le lie conceptuellement au terme d'activité, ou celui de la théorie anthropologique du didactique ${ }^{54}$ est donc pour moi discutable. Là où le modèle du jeu tente d'approcher la dynamique de l'action, son épaisseur, sa complexité, son entrelacement aux structures, l'usage du terme et de la notion de tâche peuvent la réduire, d'une manière scientifiquement impropre et éthiquement dommageable.

\section{En matière de conclusion, des critères d'usage du modèle du jeu}

Je conclurai provisoirement ici sur un point qui me semble décisif.

Un usage adéquat du jeu en tant que modèle consiste à découper au sein de l'activité humaine ce que l'on va pouvoir considérer comme les manières de jouer effectives des agents. L'activité ne se donne pas elle-même, bien sûr, comme un système de jeux, et probablement certains individus seraient-ils sponta-

54 Ibid. 
nément bien étonnés de voir leur pratique considérée comme un tel système.

La pertinence du modèle du jeu suppose que les entités que l'on identifie dans la pratique rendent adéquatement compte de la dynamique téléologique et de la structuration même de cette pratique. Il ne s'agit pas seulement de déclarer que je vois telle pratique comme un jeu que je décris de telle ou telle manière, il s'agit surtout de s'assurer que ce voir-comme a quelque pertinence, que ce voir-comme rencontre la réalité. Épineux problème, qui demande aux utilisateurs du modèle du jeu une vigilance spécifique, indissociablement épistémologique, théorique, et méthodologique. Il me semble que la détermination d'un système de critères d'usages du modèle du jeu peut aider à une telle vigilance.

Pour moi, la conception générale de tels critères repose en particulier sur la mise en regard de la modélisation avec le sens de l'action tel qu'il est vécu par les agents de cette action. La structure tripartite du modèle du jeu, que j’ai présentée ci-dessus, me semble pouvoir y aider.

L'agent devra pouvoir d'abord se reconnaître (reconnaître la nature profonde de son activité) dans le descripteur dense obtenu par la dénomination du jeu que lui présentera l'analyste. Pour reprendre le petit exemple de la lecture évoqué ci-dessus, il devra pouvoir donner son assentiment à une description de son activité du genre " ce que vous cherchez à faire, c'est commenter en connaisseur tel passage du livre ".

L'agent de l'action devra également pouvoir se reconnaître dans les règles définitoires conjecturées par l'analyste. Par exemple, il devra pouvoir trouver pertinente une règle du type " quand on commente en connaisseur un passage de livre, on doit pouvoir établir des relations sémantiques adéquates entre ce passage et d'autres passages du même livre ".

L'agent devra également pouvoir se reconnaître dans des règles stratégiques proposées par l'analyste, du type, pour l'exemple considéré, " pour établir des relations sémantiques adéquates entre divers passages du même livre, il faut pouvoir retrouver le 
système intentionnel de l'auteur ".

Enfin, dans le débat entre le chercheur et l'agent, l'expression des stratégies effectives que l'agent produit devra pouvoir être reconnue par le chercheur, jusqu’à être explicitée et partagée dans le dialogue. Agent et chercheur pourront même produire des anticipations conjointes de stratégies face à un problème donné de la pratique.

On saisit donc ici que la question méthodologique sera cruciale, et que des formes d'enquête relatives aux systèmes stratégiques des acteurs, par exemple inspirées des méthodes d'auto-confrontation ou d'instruction au sosie ${ }^{55}$, devront être systématiquement développées, ce qui suppose une instrumentation nouvelle en SHS, fondée en particulier sur le film d'étude ${ }^{56}$.

On le voit, dans ce qui précède, rencontrer la réalité, pour un modèle du jeu, suppose retrouver le sens de l'action pour l'agent. Précisons pour terminer que la pertinence scientifique ne se limite pas à cette nécessité, puisque on postulera certaines dimensions de leur pratique opaque aux agents eux-mêmes. C'est bien le sens d'une théorie scientifique, dans les sciences de l'homme et de la société, de ne pas se limiter au sens de l'action pour les individus, tout en l'intégrant à ses élaborations.

55 Yves Clot, Le Travail sans l'homme? Pour une psychologie des milieux de travail et de vie, Paris, La Découverte, 2008.

56 Cf. Andrée Tiberghien et Gérard Sensevy, «Video studies: Time and Duration in the Teaching-Learning Processes ", dans Justin Dillon et Doris Jorde (dir.), Handbook "The World of Science Education", volume 4, à paraitre, Rotterdam/ Boston/Taipei, Sense Publishers, 2012. 


\section{Bibliographie}

Bourdieu, Pierre, Choses dites, Paris, Minuit, 1987.

Bourdieu, Pierre et Loïc J. D. Wacquant, Réponses : pour une anthropologie réflexive, Paris, Seuil, 1992.

Blumer, Herbert, George Herbert Mead and Human Conduct, Walnut Creek, AltaMira Press, 2004.

Cartwright, Nancy, The Dappled World: A Study of the Boundaries of Science, Cambridge, Cambridge University Press, 1999.

Chauvier, Stéphane, Qu'est-ce qu'un jeu?, Paris, Vrin, 2007.

Chevallard, Yves, "Éducation et didactique: la tension essentielle ", Education et didactique, vol. 1, $\mathrm{n}^{\circ}$ 1, 2007, p. 9-28.

Culioli, Antoine, Variations sur la linguistique. Entretiens avec Frédéric Fau, Paris, Klincksieck, 2009.

Clot, Yves, Le Travail sans l'homme? Pour une psychologie des milieux de travail et de vie, Paris, La Découverte, 2008.

Descombes, Vincent, Le Complément de sujet, Paris, Gallimard, 2004.

Dewey, John, Logique. Théorie de l'enquête, Paris, Presses universitaires de France, 1993.

Engeström, Yrjo, "L'Interactivité orientée-objet: vers une compréhension de l'intentionnalité collective dans les activités distribuées ", dans Jean Marie Barbier et Marc Durand (dir.), Sujets, activités, environnements: approches transverses, Paris, Presses universitaires de France, 2006, p. 135-173.

Fleck, Ludvick, Genèse et développement d'un fait scientifique, Paris, Les Belles lettres, 2005.

Gallie, Walter B., Philosophy and the Historical Understanding, Londres, Chatto and Windus, 1964.

Gruson, Brigitte, Dominique Forest et Monique Loquet, Jeux de savoir, Rennes, Presses universitaires de Rennes, 2012.

Hacking, Ian, Concevoir et expérimenter, Paris, Christian Bourgois, 1989.

Harre, Rom et Luk Van Langenhove, Positioning Theory: Moral Contexts of Intentional Action, Londres, Blackwell, 1999.

Hintikka, Jaako, Fondements d'une théorie du langage, Paris, Presses universitaires de France, 1994.

Hintikka, Jaako et Gabriel Sandu, "What Is Logic? ”, dans Gabbay Dan, Paul Thagard et Paul Woods, Handbook of the Philosophy of Science, volume 5, Londres, Elsevier, 2006. p. 13-38.

James, William, dans H. Standish Thayer (dir.), Pragmatism. The Classic 
Writings, London, Hackett, 1982, p. 123-251.

Kuhn, Thomas, La Tension essentielle: tradition et changement dans les sciences, Paris, Gallimard, 1990.

Lahire, Bernard, La Condition littéraire. La double vie des écrivains, Paris, La Découverte, 2006.

Lahire, Bernard, L'Homme pluriel : les ressorts de l'action, Paris, Hachette, 2007.

Laugier, Sandra et Christiane Chauviré, Lire les Recherches Philosophiques de Wittgenstein, Paris, Vrin, 2006.

Laugier, Sandra, "L'Éducation des adultes comme philosophie morale ", Education et Didactique, vol. 5, n 3, 2011, p. 135-144.

Leibniz, Gottfried Wilhelm, Opuscules philosophiques choisis, Paris, Vrin, 1954.

Lessing, Gotthold E., Traités sur la fable, Paris, Vrin, 2008.

Lewis, David, Convention, Oxford, Blackwell, 1965.

Mead, Georges H., L'Esprit, le soi et la société, Paris, Presses universitaires de France, 2006.

Merleau-Ponty, Maurice, Éloge de la philosophie et autres essais, Paris, Gallimard, 1962.

Ricoeur, Paul, La Métaphore vive, Paris, Seuil, 1990.

Ryle, Gilbert, The Concept of Mind, London, Hutchinson's University Library, 1949.

Ryle, Gilbert, "Logic Lane. Collected Essays ", volume 2, Londres, Routledge, 2009.

Searle, John, "What Is an Institution? ", Journal of Institutional Economics, vol. $1, \mathrm{n}^{\circ} 1,2005$, p. 1-22.

Sensevy, Gérard, "Overcoming Fragmentation: Towards a Joint Action Theory in Didactics ", dans Hudson Brian et Meinert A. Meyer (dir.), Beyond Fragmentation: Didactics, Learning and Teaching in Europe, Opladen and Farmington Hills, Barbara Budrich, 2011, p. 60-76.

Sensevy, Gérard, «Patterns of Didactic Intentions. Thought Collective and Documentation Work ", dans Ghislaine Gueudet, Birgit Pepin et Luc Trouche (dir.), From Text to "Lived " Resources: Mathematics Curriculum Materials and Teacher Development, New York, Springer, 2011, p. 43-58.

Sensevy, Gérard, Le Sens du Savoir. Eléments pour une théorie de l'action conjointe en didactique, Bruxelles, De Boeck, 2011.

Sensevy, Gérard et Alain Mercier, Agir ensemble. L'action didactique conjointe du professeur et des élèves, Rennes, Presses universitaires de 
Rennes, 2007.

Sensevy, Gérard, Andrée Tiberghien, Jérôme Santini, Sylvain Laubé et Peter

Griggs, "An Epistemological Approach to Modelling: Cases Studies and Implications for Science Teaching ", Science Education, vol. 92, $\mathrm{n}^{\circ} 2$, 2008, p. 424-446.

Skyrms, Brian, Evolution of the Social Contract, Cambridge, Cambridge University Press, 1996.

Skyrms, Brian, The Stag Hunt and the Evolution of Social Structure, Cambridge, Cambridge University Press, 2004.

Thayer, H. Standish, Pragmatism. The Classic Writings, London, Hackett, 1982.

Tiberghien, Andrée et Gérard Sensevy, «Video studies: Time and Duration in the Teaching-Learning Processes ", dans Justin Dillon et Doris Jorde (dir.), Handbook "The World of Science Education", volume 4, à paraitre, Rotterdam/Boston/Taipei, Sense Publishers, 2012.

Tomasello, Michael, Origins of Human Communication, Cambridge, MIT Press, 2008.

Tomasello, Michael, Pourquoi nous coopérons, Rennes, Presses universitaires de Rennes, 2012.

Torra-Mattenklott, Catherine, "The Fable as Figure : Christian Wolff's Geometric Fable Theory and Its Creative Reception by Lessing and Herder ", Science in Context, vol. 18, no 4, 2005, p. 525-552.

Van Fraassen, Baas, Scientific Representation: Paradoxes of Perspective, Oxford, Oxford University Press, 2008.

Veyne, Paul, Comment on écrit L'histoire, Paris, Seuil, 1979.

Wittgenstein, Ludwig, Recherches philosophiques, Paris, Gallimard, 2004. 